\title{
OPEN Improvement of ligand-free modification strategy to obtain water-stable up-converting nanoparticles with bright emission and high reaction yield
}

\author{
Natalia Jurga, Dominika Przybylska, Piotr Kamiński \& Tomasz Grzyb ${ }^{\bowtie}$
}

Water-dispersible up-converting nanoparticles (UCNPs) are known to be very effective in biomedical applications. Research groups have paid special attention to the synthesis of hydrophilic UCNPs with good physicochemical properties. Being aware of this, we decided to improve the ligand-free modification method of $\mathrm{OA}_{\text {-capped }} \mathrm{NaYF}_{4}: \mathrm{Yb}^{3+}, \mathrm{Er}^{3+} / \mathrm{NaYF}_{4} \mathrm{UCNPs}$ prepared by precipitation in highboiling-point solvents as the thus-far reported methods do not provide satisfactory results. Different molarities of hydrochloric acid and various mixing times were selected to remove the organic ligand from the NPs' surface and to discover the most promising modification approach. Highly water-stable colloids were obtained with a very high reaction yield of up to $96 \%$. Moreover, the acid treatment did not affect the morphology and the size of the product. All of the crystals exhibited a bright up-conversion emission under 975 -nm excitation, which confirmed the two-photon excitation and effective energy transfer between the used dopant ions. Thus, we could establish the most successful ligand-free modification procedure.

The lanthanide $\left(\mathrm{Ln}^{3+}\right)$-doped up-converting nanoparticles (UCNPs) are interesting materials because of their specific optical properties, i.e., long luminescence lifetimes and the sharp emission bands related to the $\mathrm{Ln}^{3+}$ electronic configuration ${ }^{1}$. In the up-conversion (UC) process, the absorption of two or more near-infrared (NIR) photons is followed by the emission of light in the UV and the visible range ${ }^{2}$. The system consisting of two dopant ions $\mathrm{Yb}^{3+} / \mathrm{Er}^{3+}$ is perfect to study one of the UC mechanisms called energy transfer, in which the energy is absorbed by the sensitizer ( $\mathrm{Yb}^{3+}$ ions) and transferred to the activator $\left(\mathrm{Er}^{3+} \text { ions }\right)^{3}$. By changing the dopant-ion combination and concentration, we can observe the multi-color UC emission ${ }^{4,5}$. Note that using NIR light as an excitation source is beneficial because of the deep penetration depth in tissues, small photodamage to living organisms, and the absence of autofluorescence from cells ${ }^{1,6}$.

The appropriate selection of UCNPs' host matrix plays an important role in achieving the desired luminescent properties ${ }^{7}$. The commonly used hosts of the UC materials are fluorides because of their excellent chemical stability and low phonon energy. The obtainment of $\mathrm{Ln}^{3+}$-doped $\mathrm{NaYF}_{4}$ with a hexagonal structure is particularly beneficial, as it is considered to be the most profitable UC luminophore ${ }^{8}$. Additionally, the UC efficiency can be enhanced by the presence of the inert shell, which protects luminescent ions in the core from the non-radiative transitions ${ }^{9}$. The high-quality UCNPs with a particular size, shape, and phase can be synthesized using precipitation in the high-boiling-point solvent method. During the synthesis, selected rare earth (RE) ion precursors are decomposed to form the desired UCNPs by controlling the temperature, pressure, and solvent composition ${ }^{10}$. Unfortunately, the synthesized oleate-capped (OA-capped) UCNPs cannot be used in tissue and cell experiments because of their insolubility in water ${ }^{11}$. Therefore, it is advantageous to modify their surface ${ }^{12,13}$.

Water is the main component of living organisms. Therefore, the hydrophilic NPs are extremely attractive candidates for biomedical applications such as bioimaging, photodynamic therapy, drug delivery, cellular nanothermometry, harmful ion sensors, and cancer diagnosis and treatment ${ }^{14-20}$. Regrettably, the NPs dispersed in water show decreased luminescence intensity as compared to those dispersed in organic solvents. This phenomenon is observed because of the quenching related to the high vibrational levels of hydroxyl groups ${ }^{21}$. 
It is possible to distinguish several strategies of modifying the NP surface, such as layer-by-layer assembly, ligand exchange, ligand oxidation, ligand-free modification, and silanization ${ }^{22}$. The ligand-free modification method of the NP surface described by Capobianco's research group is well known and used ${ }^{23}$. This method allows the preparation of water-dispersible $\mathrm{NaYF}_{4}$ doped with $\mathrm{Ln}^{3+}$ ions with high UC emission intensity. As a result of the acid treatment $(\mathrm{pH}=4)$, the oleate ligands are removed from the surface of the OA-capped UCNPs. The modified NPs are stable in aqueous solutions and ready for further surface conjugation with biomolecules ${ }^{22,24}$. This approach can also be used in other host materials ${ }^{18,25,26}$. Thus far, many research groups have obtained ligandfree materials according to the described procedure, often making small changes. For instance, Guo et al. and Liu et al. decided to change the molarity of the used hydrochloric acid ${ }^{21,27}$. It is worth mentioning that $\mathrm{pH}$ values are selected depending on the particle size, e.g. $\mathrm{pH}<3$ are reported for $\beta$-phase crystals smaller than $30 \mathrm{~nm}^{27}$. Other research groups changed the ultrasonication time, as well as the time of mixing the NPs with acid ${ }^{20,28,29}$. Note that the described method can be associated with difficulties, such as the tendency of NPs to agglomerate. Therefore, the yield of this modification is usually only in the range of $30-35 \%{ }^{24}$. Moreover, the acidic environment can lead to $\alpha-\mathrm{NaREF}_{4}$ dissolution followed by precipitation of $\mathrm{REF}_{3}$. Fortunately, the phase transformation is not observed for the thermodynamically stable $\beta$-phase $\mathrm{NaREF}_{4}{ }^{27}$.

The main goal of our study was to improve the ligand-free modification method of OA-capped UCNPs synthesized using precipitation in high-boiling-point solvents. We developed a novel procedure to obtain bare core/ shell $\mathrm{NaYF}_{4}: \mathrm{Yb}^{3+}, \mathrm{Er}^{3+} / \mathrm{NaYF}_{4} \mathrm{UCNPs}$ with a high reaction yield of up to $96 \%$. Different molarities of hydrochloric acid and various reaction times were used to transfer the product from an organic to an aqueous solution: $2 \mathrm{M} / 2 \mathrm{~h}, 0.1 \mathrm{M} / 2 \mathrm{~h}$, and $2 \mathrm{M} / 15 \mathrm{~min}$, respectively. The effects of the changing modification conditions on the reaction yield, UCNP morphology, and spectroscopic properties were demonstrated. Furthermore, the stability of water-dispersible UC nanomaterials was confirmed.

\begin{abstract}
Materials and methods
Materials. The $\mathrm{RE}$ oxides $\mathrm{Y}_{2} \mathrm{O}_{3}$ (99.99\%), $\mathrm{Er}_{2} \mathrm{O}_{3}$ (99.99\%), and $\mathrm{Yb}_{2} \mathrm{O}_{3}$ (99.99\%) were purchased from Stanford Materials. Ethanol (99.8\%), acetic acid glacial (99.5\%), and acetic acid (80\%) were obtained from POCH S.A; oleic acid (90\%) and 1-octadecene (90\%) from Alfa Aesar; and n-hexane ( $\geq 99 \%)$ from Honeywell. Hydrochloric acid (ultrapure, $37 \%$ ) and sodium oleate (82\%) were obtained from Sigma-Aldrich and ammonium fluoride (99\%) from ACS Reagent. The synthesis of NPs was carried out under the flow of nitrogen (99.99\%, Linde). In the surface modification process, hydrochloric acid (ultrapure) from Romil was used. The aqua solutions of hydrochloric acid were prepared by the dissolution of the above-mentioned ultrapure hydrochloric acid in distilled water.
\end{abstract}

Synthesis of $\mathrm{OA}$-capped $\mathrm{NaYF}_{4}: \mathrm{Yb}^{3+}, \mathrm{Er}^{3+} / \mathrm{NaYF}_{4}$ core/shell up-converting nanoparticles. The core-shell $\mathrm{NaYF}_{4}: \mathrm{Yb}^{3+}, \mathrm{Er}^{3+} / \mathrm{NaYF}_{4}$ nanomaterials were synthesized using precipitation in high-boiling-point solvents $^{30,31}$. Two different RE ion precursors (RE acetates and RE chlorides) were used to obtain materials with the desired size. The approach was based on the heating of RE ion precursors in the mixture of highboiling-point solvents oleic acid and 1-octadecene (1:1 ratio), after their earlier outgassing under low pressure $\left(<10^{-1} \mathrm{mbar}\right)$. A detailed description of the procedure is presented in Supporting Information.

Synthesis of ligand-free $\mathrm{NaYF}_{4}: \mathrm{Yb}^{3+}, \mathrm{Er}^{3+} / \mathrm{NaYF}_{4}$ core/shell up-converting nanoparticles. The ligand-free $\mathrm{NaYF}_{4}: \mathrm{Yb}^{3+}, \mathrm{Er}^{3+} / \mathrm{NaYF}_{4} \mathrm{NPs}$ were prepared through the acidic treatment of the OA-capped UCNPs by using the procedure reported by Bogdan et al. with some modifications ${ }^{23}$. Firstly, $100 \mathrm{mg}$ of the appropriate OA-capped NPs were dispersed in $5 \mathrm{~mL}$ of $\mathrm{n}$-hexane, resulting in the NPs concentration of $20 \mathrm{mg} / \mathrm{mL}$. To this suspension, $2.5 \mathrm{~mL}$ of the water solution of hydrochloric acid $(2 \mathrm{M}$ or $0.1 \mathrm{M})$ was added to protonate the oleate ligands, which led to removal of the oleic acid from the NPs' surface. The flasks were closed, and the mixtures were vigorously stirred at room temperature $(2 \mathrm{~h}$ or $15 \mathrm{~min})$. On the basis of a series of laboratory experiments, three different combinations of $\mathrm{HCl}$ molarity and mixing time were chosen and reported in this article: $2 \mathrm{M} / 2 \mathrm{~h}$, $0.1 \mathrm{M} / 2 \mathrm{~h}$, and $2 \mathrm{M} / 15 \mathrm{~min}$, respectively. Note that the reaction time in the last experiment was decreased to $15 \mathrm{~min}$ because after that time, the organic solvent became transparent and the DLS analysis confirmed the absence of UCNPs in the organic phase. Therefore, it was assumed that the particles were successfully transferred to the aqueous solution. Next, the mixtures were ultrasonicated for $5 \mathrm{~min}$ and transferred to a centrifuge tube. The products were collected by centrifugation at $9000 \mathrm{rpm}$ for $15 \mathrm{~min}$ and washed with a mixture of water and ethanol (in the ratio of 1:1) to discard organic layer containing the oleic acid molecules. The washing procedure was repeated two times. The bare UCNPs were dispersed in distilled water and stored at $4{ }^{\circ} \mathrm{C}$. A schematic representation of the synthesis and surface modification of OA-capped UCNPs is presented in Fig. 1.

Characterization of materials and instrumentation. The structure of OA-capped nanomaterials was characterized by an X-ray diffraction (XRD) powder measurement using a Bruker AXS D8 Advance X-ray diffractometer with $\mathrm{Cu} \mathrm{Ka}$ radiation $(\lambda=0.15406 \mathrm{~nm})$. The morphology and the size of the products were determined by transmission electron microscopy (TEM) using an HT7700 electron microscope operating at 40 or $120 \mathrm{kV}$. The hydrodynamic diameter, polydispersity index and the zeta potential of UCNPs were measured in n-hexane (OA-capped NPs; $0.25 \mathrm{mg} / \mathrm{mL}$ ) and the water solution (bare NPs; $0.25 \mathrm{mg} / \mathrm{mL}$ ) at $25{ }^{\circ} \mathrm{C}$ by Zetasizer Nano ZS from Malvern equipped with a laser with 632.8-nm wavelength and using backward scattering $\left(173^{\circ}\right)$. A thermogravimetric (TGA) analysis was performed using Thermogravimetric Analyzer TGA 4000 (Perkin Elmer). The measurement was conducted in the temperature range of $30-600{ }^{\circ} \mathrm{C}$ (heating rate: $10^{\circ} \mathrm{C} /$ min). Infrared spectra were registered for samples in KBr pellets using JASCO 4200 FT-IR spectrophotometer. The spectroscopic properties of the OA-capped nanomaterials dispersed in $n$-hexane $(1 \mathrm{mg} / \mathrm{mL})$ and the bare 


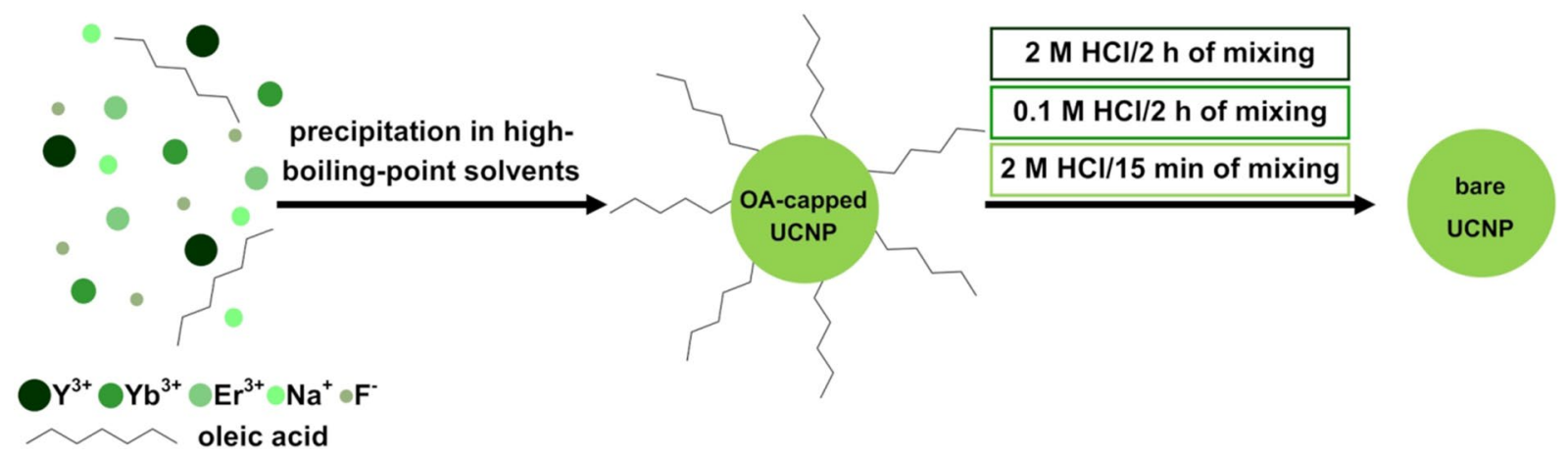

Figure 1. Schematic representation of the synthesis and ligand-free modification of OA-capped $\mathrm{NaYF}_{4}: \mathrm{Yb}^{3+}, \mathrm{Er}^{3+} / \mathrm{NaYF}_{4}$.

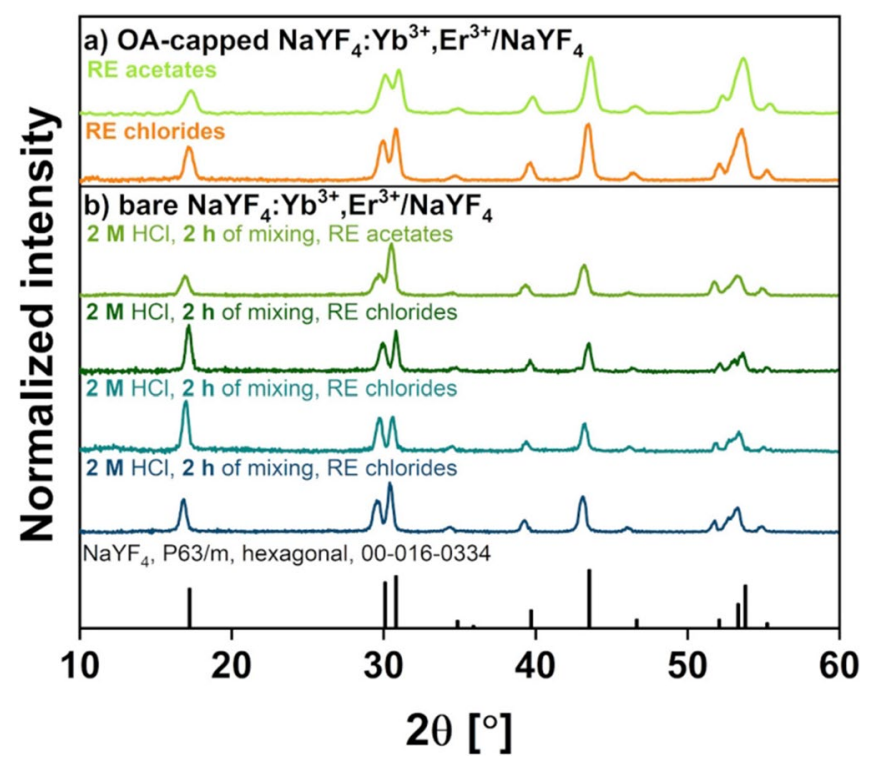

Figure 2. XRD patterns of (a) OA-capped and (b) ligand-free core/shell $\mathrm{NaYF}_{4}: \mathrm{Yb}^{3+}, \mathrm{Er}^{3+} / \mathrm{NaYF}_{4} \mathrm{UCNPs}$, and the XRD reference pattern from the JCPDS Database 00-016-0334.

UCNPs dispersed in water $(1 \mathrm{mg} / \mathrm{mL})$ were registered at room temperature using a fiber-coupled CNI $2 \mathrm{~W}$ continuous wave (CW) diode laser with 808-, 975-, 1208-, and 1532-nm wavelengths equipped with PIXIS:256E Digital CCD Camera with SP-2156 Imaging Spectrograph (Princeton Instruments). The NPs were excited with the abovementioned CW laser using $\lambda_{\text {exc }}=975 \mathrm{~nm}$.

\section{Results and discussion}

The core/shell UCNPs were synthesized by precipitation in high-boiling-point solvents. Directly after the NPs' synthesis, the structure and the phase purity of the OA-capped products obtained from RE acetates and RE chlorides were verified using an XRD analysis (Fig. 2). The $\mathrm{NaYF}_{4}: \mathrm{Yb}^{3+}, \mathrm{Er}^{3+} / \mathrm{NaYF}_{4}$ crystals exhibited a hexagonal phase, which was consistent with the reference pattern JCPDS 00-016-0334. The registered broad reflections confirmed that the products were nano-sized and well crystallized. Moreover, no additional impurity peaks were observed. The XRD analysis was also performed for the ligand-free UCNPs. Importantly, no phase and structure transformations were registered after the acid treatment of UCNPs.

The size and the morphology of the obtained products were determined using a TEM analysis (Fig. 3). Both the OA-capped and the ligand-free NPs had an average size of less than $30 \mathrm{~nm}$ and a narrow size distribution, which indicated that the obtained materials were of good quality. Additionally, the UCNPs did not agglomerate even after the removal of ligands from their surface. This suggests that the positively charged bare $\mathrm{Ln}^{3+}$-UCNPs were surrounded by the water molecules, which prevented their agglomeration. Firstly, the materials were obtained using RE acetates. The products were in the form of small rods with $21.9 \pm 1.3 \mathrm{~nm}$ in length and $14.7 \pm 0.8 \mathrm{~nm}$ in width (Fig. 3a). The TEM pictures were also collected after the surface modification of the OA-capped NPs. Their average size was decreased $(12.5 \pm 0.8 \mathrm{~nm})$, and the shape changed to hexagonal (Fig. 3b). Long-term exposure of NPs to $\mathrm{HCl}$ solution causes the shell etching and is usually followed by increasing number of surface defects ${ }^{32}$. Such weakened ligand-free NPs are more susceptible to the dissolution process, which leads 


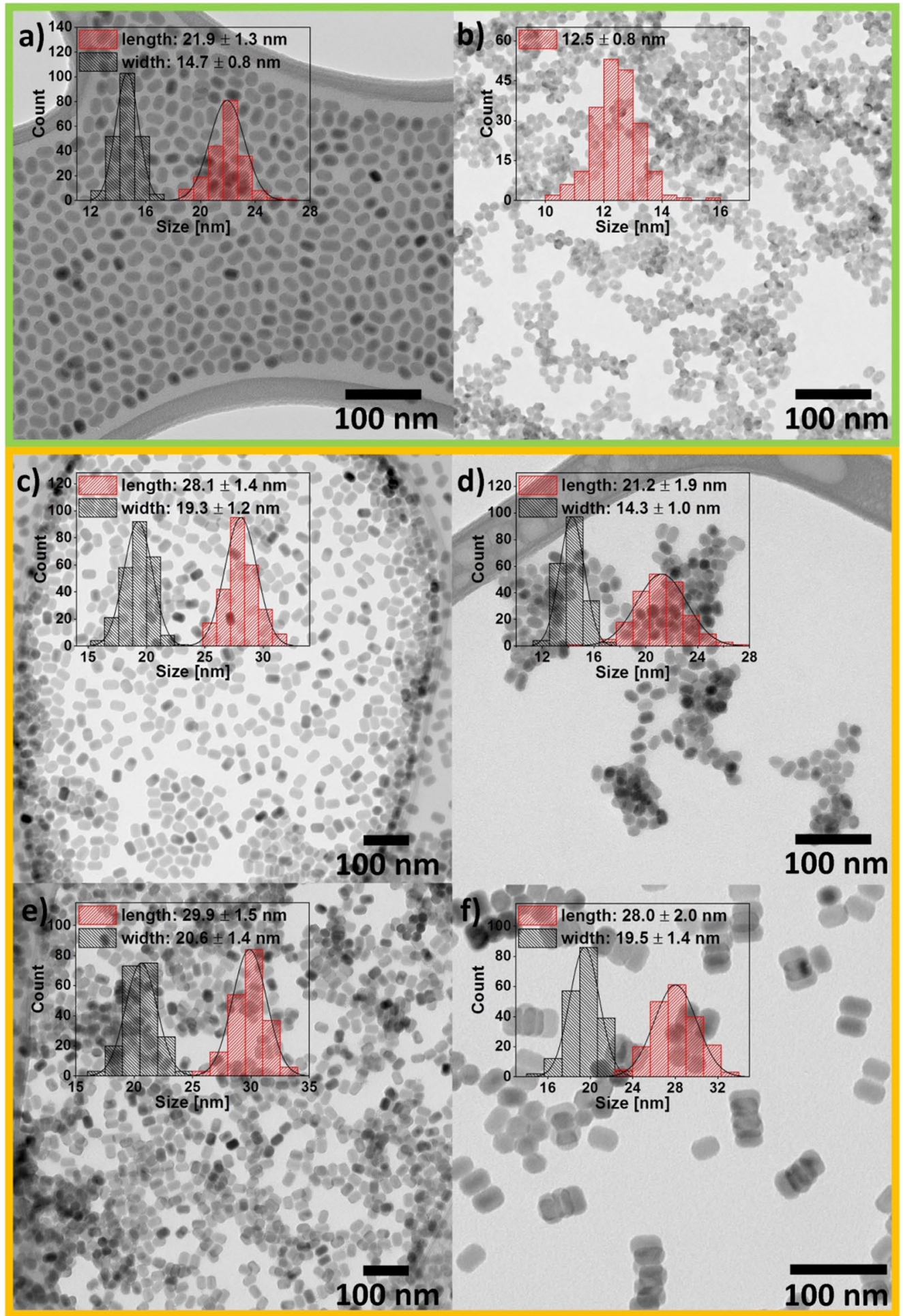

Figure 3. TEM images and particle size distribution of (a,c) OA-capped and (b,d,e,f) ligand-free $\mathrm{NaYF}_{4}: \mathrm{Yb}^{3+}, \mathrm{Er}^{3+} / \mathrm{NaYF}_{4}$ UCNPs. Different modification conditions were used (b,d) $2 \mathrm{M} / 2 \mathrm{~h},(\mathbf{e}) 0.1 \mathrm{M} / 2 \mathrm{~h}$, and (f) $2 \mathrm{M} / 15 \mathrm{~min}$, which denote the $\mathrm{HCl}$ molarity and the time of mixing, respectively. Color coding is used to indicate the UCNPs obtained from the RE acetates (green) and the RE chlorides (orange).

to size reduction. After observing the effect of the modification process on the NPs' morphology and size and considering that the solubility rate depends on surface-to-volume ratio ${ }^{33}$, it was decided to obtain the OA-capped product with a slightly larger size to conduct more experiments and better illustrate the changes. On the basis 
of the laboratory experience of our research group, $\mathrm{RE}$ chlorides were chosen as the RE ion precursors. The use of RE chlorides instead of RE acetates was more beneficial, and it allowed us to prevent the production of toxic compounds during the thermolysis of trifluoroacetate ${ }^{8}$. Note that the OA-capped NPs obtained from the RE chlorides maintained the same morphology as the crystals obtained from the RE acetates but had a larger size (length: $28.1 \pm 1.4 \mathrm{~nm}$, width: $19.3 \pm 1.2 \mathrm{~nm}$; Fig. $3 \mathrm{c}$ ). Similar to the previously conducted experiment, the selected reaction conditions (2- $\mathrm{M} \mathrm{HCl}$ and $2 \mathrm{~h}$ of mixing) reduced the size of the bare UCNPs (length: $21.2 \pm 1.9 \mathrm{~nm}$, width: $14.3 \pm 1.0 \mathrm{~nm}$; Fig. $3 \mathrm{~d}$ ) in comparison to the OA-capped crystals. To prevent this phenomenon, the molarity of the used acid and the stirring time were modified, respectively. After changing the modification conditions, the size of the bare crystals changed insignificantly, within the limits of error. The rods with a length of $29.9 \pm 1.5 \mathrm{~nm}$ and a width of $20.6 \pm 1.4 \mathrm{~nm}$ (Fig. 3e) were obtained with $0.1-\mathrm{M} \mathrm{HCl}$ and $2 \mathrm{~h}$ of stirring. In turn, the application of $2-\mathrm{M} \mathrm{HCl}$ and the reduction of the mixing time to $15 \mathrm{~min}$ allowed us to obtain NPs with a length of $28.0 \pm 2.0 \mathrm{~nm}$ and a width of $19.5 \pm 1.4 \mathrm{~nm}$ (Fig. 3f). Importantly, after the modification of the OA-capped UCNPs obtained from the RE chlorides, the morphology remained unchanged. Nevertheless, despite of the NPs size reduction, all of the bare particles retain pure hexagonal phase which was confirmed by the XRD analysis. The results show how important it is to find the appropriate mixing time that allows for the removal of ligands from the particle surface without adversely affecting the properties of the nanoparticles.

Additionally, the size distribution was determined using DLS measurements (Fig. S1). It was again confirmed that changing the RE ion precursor (from RE acetates to RE chlorides) allowed us to obtain bigger NPs. Moreover, the size of ligand-free nanomaterials was larger than that calculated from the TEM images. This was related to the fact that the DLS analysis provided information about the NPs' hydrodynamic diameter swelling in a water solution, whereas TEM determined the size of the crystals. The polydispersity index (PdI) were determined to check the NPs' size uniformity and samples' heterogeneity. It is assumed that the monodisperse NPs with narrow size distribution are characterized by the PdI values lower than 0.1 . When the PdI values are between 0.1 and 0.4 , the particles are polydisperse with a medium size distribution. The higher PdI values indicate the presence of NPs with various sizes which is not acceptable in biomedical applications ${ }^{11}$. The PdI values of OA-capped crystals obtained from RE acetates and RE chlorides were in the range of 0.07-0.09, which confirmed monodispersity of NPs. The increase of PdI values of bare crystals compared to OA-capped can be observed (PdI values in the range of $0.21-0.38$ ). However, the values still do not exceed 0.4 .

The zeta potential measurements were conducted to verify the removal of the ligands from the NPs' surface and to specify the stability of the bare nanomaterials in an aqueous solution. During UCNPs' acid treatment, the oleate ligands are protonated and dissociated from the NPs' surface leaving positively charged $\mathrm{Ln}^{3+}-\mathrm{UCNP}$. The water molecules, which are present in the aqueous solution, can surround the NPs and thereby improve colloid stability. Importantly, the $\mathrm{pH}$ of prepared water colloids was in the range of 6.3 to 6.5 , which suggests the presence of $\mathrm{H}_{3} \mathrm{O}^{+}$ions in the solutions. Therefore, it is possible to observe the repulsive electrostatic forces between two adjacent UCNPs with positive zeta potential values, which results in preventing crystals agglomeration. The smallest value of the zeta potential $(33.1 \pm 5.4 \mathrm{mV})$ had the bare NPs obtained from the RE acetates after the 2-M $\mathrm{HCl}$ treatment and $2 \mathrm{~h}$ of mixing. In the case of the ligand-free products obtained from the RE chlorides, the following zeta potential values were recorded: $39.9 \pm 5.4 \mathrm{mV}, 36.1 \pm 7.1 \mathrm{mV}$, and $39.4 \pm 6.0 \mathrm{mV}$ for the subsequent $\mathrm{HCl}$ solution molarities and the times of mixture stirring: $2 \mathrm{M} / 2 \mathrm{~h}, 0.1 \mathrm{M} / 2 \mathrm{~h}$, and $2 \mathrm{M} / 15 \mathrm{~min}$, respectively. The surface charges of all the ligand-free products were positive, which confirmed that the NPs' surface modification was successful. Interestingly, the zeta potential values were higher than $30 \mathrm{mV}$ even after six months for all of the modified crystals. This indicated the very good stability of the NPs in the water solution.

The FT-IR measurements of both OA-capped and bare crystals were carried out to confirm the dissociation of oleate ligands from the NPs' surface (Fig. S2). The FT-IR spectra of OA-capped particles showed two peaks at $2930 \mathrm{~cm}^{-1}$ and $2854 \mathrm{~cm}^{-1}$, which are attributed to the $-\mathrm{CH}_{2}$ and $-\mathrm{CH}_{3}$ stretching, respectively. Moreover, the presence of bands at $1467 \mathrm{~cm}^{-1}$ and $1557 \mathrm{~cm}^{-1}$ confirmed the asymmetric and symmetric -COO- stretches. After the ligands removal, the bands related to organic groups stretching vibrations were significantly weakened. The additional band appeared at $1630 \mathrm{~cm}^{-1}$ and is assigned to the bending mode of water molecules ${ }^{34}$.

The modification conditions were changed to obtain a stable suspension of NPs in water without the change in their morphology and size. However, achieving the highest possible modification yield, which resulted in the smallest product loss, was also very important to our research group. Conducting the 2-h synthesis with the use of the 2-M HCl solution allowed us to obtain a reaction yield in the range of $47-50 \%$. Unfortunately, the modification yield decreased to $37 \%$ with the reduction of the molarity of the hydrochloric acid solution. It can be related to the fact that the $0.1-\mathrm{M} \mathrm{HCl}$ solution was not sufficiently strong to protonate all of the OA anions; hence, not all of the particles were transferred from the organic solution to water. However, the combination of the $2-\mathrm{M} \mathrm{HCl}$ solution and $15 \mathrm{~min}$ of mixing turned out to be the most beneficial, as it increased the reaction yield up to $96 \%$. Several repetitions of this approach were performed, in which the reaction yields were in the range of $75-96 \%$. Moreover, the physicochemical properties of these ligand-free NPs were satisfactory, which was proven in this article.

The obtained materials are particularly interesting because of their unique spectroscopic properties. The UC emission spectra of the OA-capped UCNPs dispersed in n-hexane and the bare UCNPs dispersed in water were recorded using the same excitation wavelength $\left(\lambda_{\text {exc }}=975 \mathrm{~nm}\right)$, laser power density $\left(32 \mathrm{~W} / \mathrm{cm}^{2}\right)$, and NPs' concentration ( $1 \mathrm{mg} / \mathrm{mL}$; Fig. 4). On each spectrum, we observed five narrow bands related to the $\mathrm{Er}^{3+} \mathrm{elec}^{-}$ tronic transitions. The green emission color was strongly attributed to the transitions from the ${ }^{2} \mathrm{H}_{11 / 2}$ and ${ }^{4} \mathrm{~S}_{3 / 2}$ to the ${ }^{4} \mathrm{I}_{15 / 2}$ level. In turn, the transition from the ${ }^{4} \mathrm{~F}_{9 / 2}$ to the ${ }^{4} \mathrm{I}_{15 / 2}$ level was responsible for the red luminescence color. The three bands centered at 525,544, and $658 \mathrm{~nm}$ had the strongest influence on the emission color. Two other bands centered at 805 and $844 \mathrm{~nm}$ were distinguished; they were associated with the ${ }^{4} \mathrm{I}_{9 / 2} \rightarrow{ }^{4} \mathrm{I}_{15 / 2}$ and the ${ }^{4} \mathrm{~S}_{3 / 2} \rightarrow{ }^{4} \mathrm{I}_{13 / 2}$ transitions of $\mathrm{Er}^{3+}$, respectively. Note that the OA-capped product synthesized from the RE chlorides was characterized by the higher luminescence intensity than that of the product obtained from the RE acetates 


\section{$\lambda_{\text {exc }}=975 \mathrm{~nm}$; laser power density: $32 \mathrm{~W} / \mathrm{cm}^{2}$}
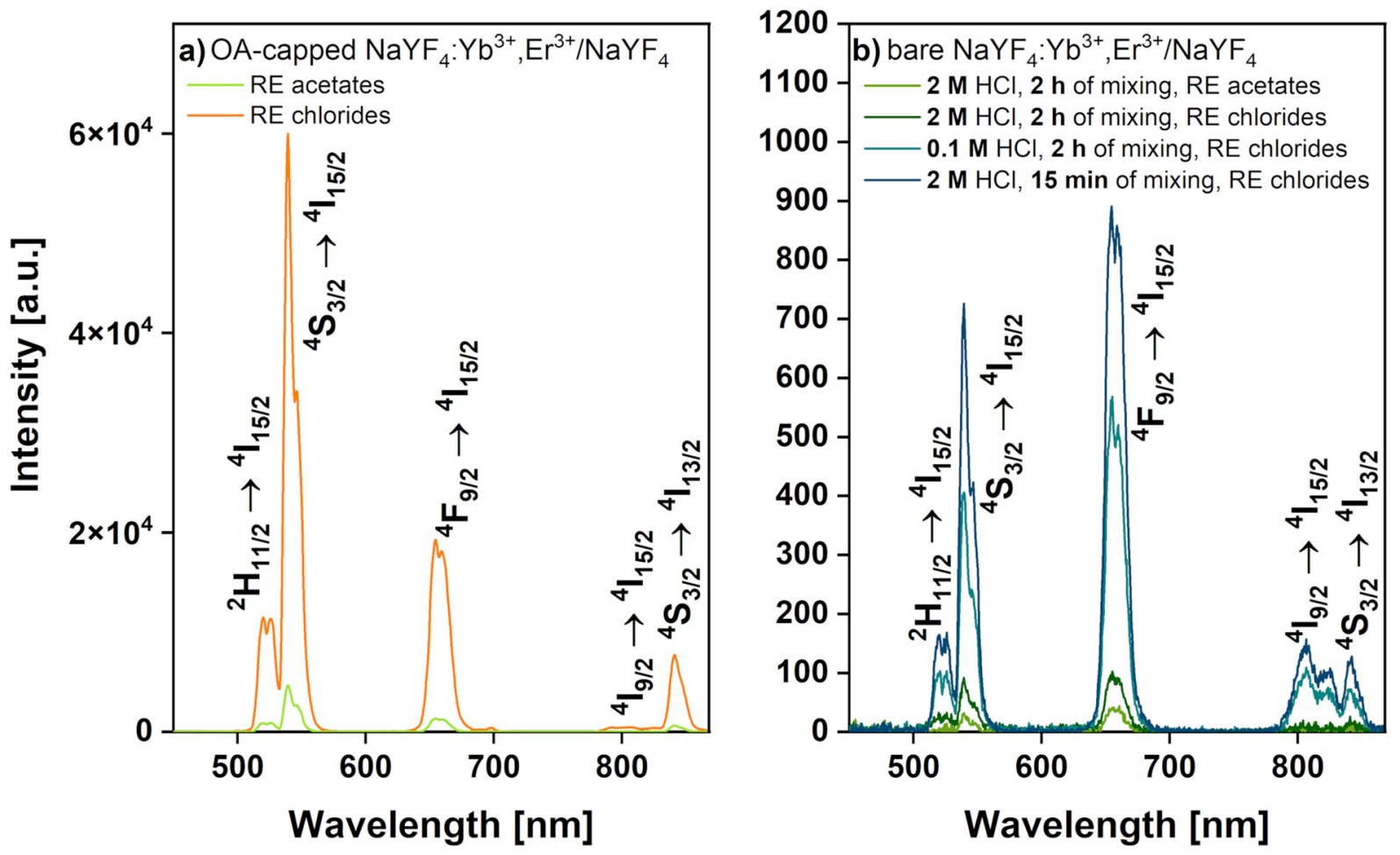

Figure 4. UC emission spectra of (a) OA-capped $\mathrm{NaYF}_{4}: \mathrm{Yb}^{3+}, \mathrm{Er}^{3+} / \mathrm{NaYF}_{4} \mathrm{UCNPs}$ obtained from $\mathrm{RE}$ acetates and RE chlorides and (b) bare UCNPs after ligand-free modification with three different combinations of $\mathrm{HCl}$ molarity and mixing time, $\lambda_{\mathrm{exc}}=975 \mathrm{~nm}$. The NPs' concentration was $1 \mathrm{mg} / \mathrm{mL}$ for both the OA-capped and the bare UCNP solutions.

(Fig. 4a). This fact could be related to the different sizes of NPs and the reduction of the surface defects in the larger crystals. The spectroscopic properties of the modified NPs were also determined (Fig. 4b). The emission intensity of ligand-free products considerably decreased in comparison to the OA-capped NPs. This phenomenon was connected to the non-radiative transfer of energy from the dopant ions to the water molecules. Note that of all the bare particles, the brightest luminescence had the product modified for 15 min with the 2-M HCl solution. Slightly worse spectroscopic properties had NPs, in which the ligands were removed by the 0.1-M acid for $2 \mathrm{~h}$. The lowest emission intensities were recorded when the NPs were treated with 2-M HCl for a long time. Therefore, it could be concluded that with the reduction of the product size, the luminescence intensity decreased. It was confirmed that the prolonged exposure of particles to acid caused visible changes in the NPs' size associated with the shell etching. Furthermore, the comparison of the peak area of the bands related to the green and red emission colors was shown. Interestingly, the green-to-red emission ratio was tuned from values close to 2.5 to values below 1 , when the products were transferred from an organic to an aqueous solution. It showed that the UC luminescence of the OA-capped crystals was dominated by the green emission color as opposed to the emission color of the bare NPs dominated by the red. This was the effect of the non-radiative depopulation of the ${ }^{2} \mathrm{H}_{11 / 2}$ and ${ }^{4} \mathrm{~S}_{3 / 2}$ states to the red-emitting ${ }^{4} \mathrm{~F}_{9 / 2}$ level caused by the $-\mathrm{OH}$ groups.

The energy-level diagram of the used dopant ions $\mathrm{Yb}^{3+} / \mathrm{Er}^{3+}$, as well as their excitation and deactivation pathways, is presented in Fig. 5a. One possible route of excitation was based on the energy transfer UC mechanism, which took place between the dopant ions. The $\mathrm{Yb}^{3+}$ ions, which were sensitizers, absorbed the energy and were excited from the ground state to the ${ }^{2} \mathrm{~F}_{5 / 2}$ level. Then, the neighboring $\mathrm{Er}^{3+}$ ions called activators could be excited to the ${ }^{4} \mathrm{I}_{11 / 2}$ level by the energy transfer from the $\mathrm{Yb}^{3+}$ ions. Another photon could be absorbed, resulting in the subsequent $\mathrm{Er}^{3+}$ excitation to the ${ }^{4} \mathrm{~F}_{7 / 2}$ level. Consequently, it was possible to observe non-radiative relaxation to the ${ }^{2} \mathrm{H}_{11 / 2},{ }^{4} \mathrm{~S}_{3 / 2},{ }^{4} \mathrm{~F}_{9 / 2}$, and ${ }^{4} \mathrm{I}_{9 / 2}$ levels (dotted arrows), as well as the UC emission (solid arrows) responsible for the different luminescence colors. Another possible excitation route was the excited-state absorption mechanism occurring from the ${ }^{4} \mathrm{I}_{13 / 2}$ to the higher ${ }^{4} \mathrm{~F}_{9 / 2} \mathrm{Er}^{3+}$ state. This route, as well as the energy transfer assisted by the $\mathrm{Yb}^{3+}$ ions, led to the enhancement of the red emission related to the feeding of the ${ }^{4} \mathrm{~F}_{9 / 2}$ level. Note that in the case of the NPs dispersed in an aqueous solution, the solvent played a critical role in the reduction of the UC luminescence intensity of the NPs? ${ }^{9}$. The water quenching (wavy arrows) affected all the energy levels involved in the NIR to the visible UC mechanism. This could be attributed to the absorption of water near $980 \mathrm{~nm}$, which could debilitate the excitation light ${ }^{21}$. Additionally, the water molecules could extract energy from the excited dopant ions. The energy gap between the ${ }^{4} \mathrm{I}_{11 / 2}$ and the ${ }^{4} \mathrm{I}_{13 / 2}$ levels of $\mathrm{Er}^{3+}$ well matched with the hydroxyl phonon energy $\left(\sim 3400 \mathrm{~cm}^{-1}\right)$. Because of this fact, the ${ }^{4} \mathrm{I}_{11 / 2} \rightarrow{ }^{4} \mathrm{I}_{13 / 2}$ relaxation could be intensified through a cross-energy 

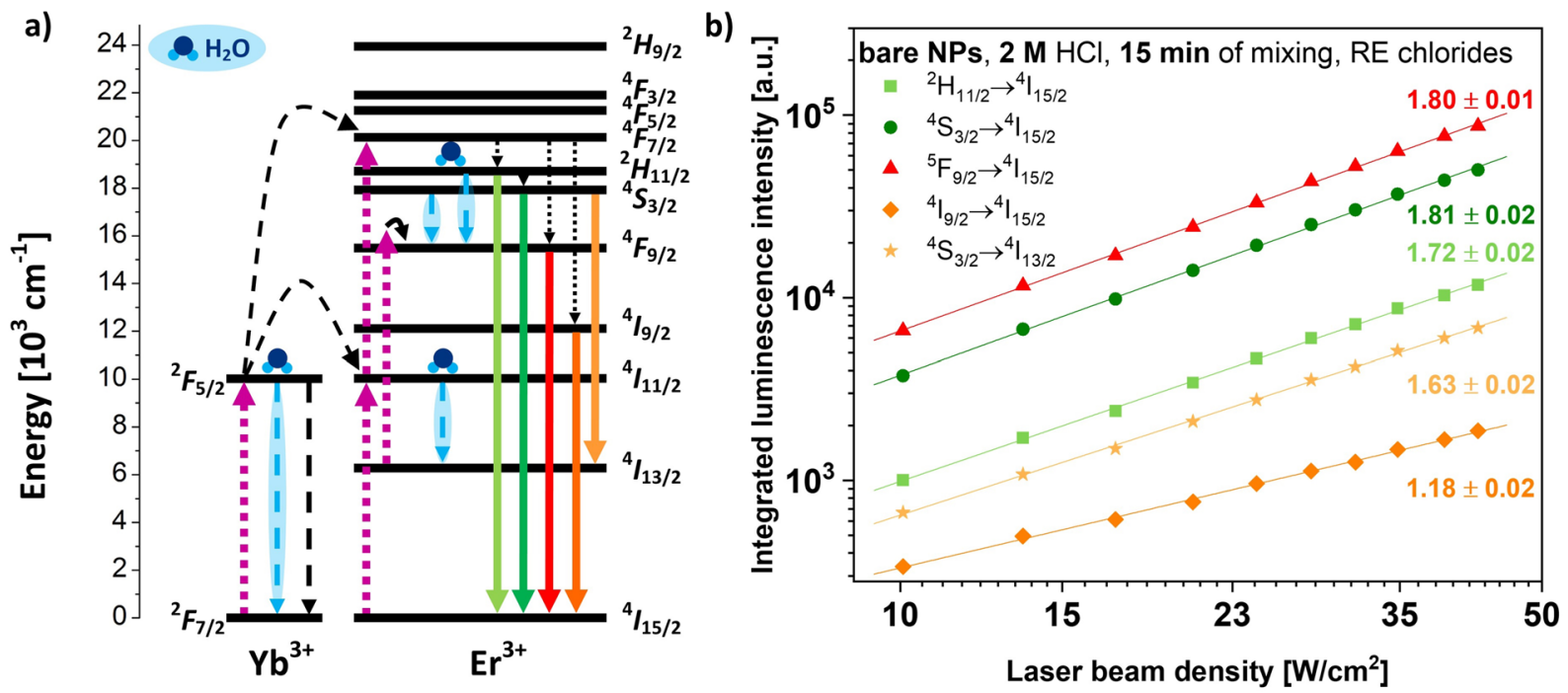

Figure 5. (a) Energy-level diagram for $\mathrm{Yb}^{3+}$ and $\mathrm{Er}^{3+}$ ions as well as proposed up-conversion mechanism (including de-excitation by water molecules) and (b) power-dependent slope values for $\mathrm{Er}^{3+}$ transitions in ligand-free $\mathrm{NaYF}_{4}: \mathrm{Yb}^{3+}, \mathrm{Er}^{3+} / \mathrm{NaYF}_{4}$ obtained from RE chlorides and modified for 15 min by using 2-M $\mathrm{HCl}$ solution.

transfer to the water particles. The $\mathrm{O}-\mathrm{H}$ vibrations also had an impact on the non-radiative decays of the ${ }^{4} \mathrm{~S}_{3 / 2}$ and the ${ }^{2} \mathrm{H}_{11 / 2}$ levels of $\mathrm{Er}^{3+35}$.

In the UC process, the observed luminescence intensity $\left(I_{U C}\right)$ was proportional to the excitation laser power $(P): I_{U C} \propto P^{n}$, where the $n$ symbol represents the number of photons involved in the UC mechanism ${ }^{36}$. Therefore, the dependencies of the integral luminescence intensity on the laser beam density (Fig. 5b) were studied for the water-stable UCNPs characterized by the brightest UC emission. The UC emission intensities were measured using the 975-nm excitation wavelength and a laser beam density ranging from 10 to $43 \mathrm{~W} / \mathrm{cm}^{2}$. Linear fitting with high coefficients of determination $R^{2}(\leq 0.998)$ allowed us to calculate the slope values for five $\mathrm{Er}^{3+}$ electronic transitions: from ${ }^{2} \mathrm{H}_{11 / 2},{ }^{4} \mathrm{~S}_{3 / 2},{ }^{5} \mathrm{~F}_{9 / 2}$ and ${ }^{4} \mathrm{I}_{9 / 2}$ to ${ }^{4} \mathrm{I}_{15 / 2}$, as well as from ${ }^{4} \mathrm{~S}_{3 / 2}$ to the ${ }^{4} \mathrm{I}_{13 / 2}$ level. They were in the range of one to two (err $\leq 0.02$ ), suggesting a two-photon excitation process, which was consistent with the proposed UC mechanism (Fig. 5a). Note that the observed slope values were not ideal integers. This phenomenon was related to the presence of various excitation possibilities, as well as the non-radiative decays of the $\mathrm{Er}^{3+}$ intermediate excited states ${ }^{37}$.

By comparing three different combinations of ligand-free modification conditions, we could clearly distinguish the best approach for removing the organic ligands from the NPs' surface. The above-described results of the zeta potential measurements, the green-to-red emission ratios, surface modification yields, DLS measurements, and TEM analyses for both the OA-capped and the bare $\mathrm{NaYF}_{4}: \mathrm{Yb}^{3+}, \mathrm{Er}^{3+} / \mathrm{NaYF}_{4}$ particles are shown in Fig. 6 to help the reader distinguish the differences between the modified crystals. Additionally, the ligand-free NPs characterized by the best physicochemical properties and the highest ligand removal reaction yield were marked with the extra red circle.

\section{Conclusions}

To conclude, an improved procedure for obtaining the ligand-free $\mathrm{NaYF}_{4}: \mathrm{Yb}^{3+}, \mathrm{Er}^{3+} / \mathrm{NaYF}_{4} \mathrm{UCNPs}_{\text {was }}$ developed. Three different combinations of modification conditions based on the changes in the acid molarity and the mixing time were tested to confirm their impact on the physicochemical properties of the NPs. The hexagonal OA-capped crystals were synthesized using precipitation in high-boiling-point solvents. Additionally, the organic ligand removal was performed successfully by using the $\mathrm{HCl}$ solution. The procedure based on the use of the $2-\mathrm{M} \mathrm{HCl}$ solution and $15 \mathrm{~min}$ of mixing was the most effective of all of the tested approaches, as it enabled us to achieve the highest reaction yield of up to $96 \%$. Importantly, in comparison to those of the hydrophobic NPs, the size and the shape of the bare crystals modified according to this approach remained unchanged. The small UCNPs were stable in the water solution even after six months, which made it possible for us to apply them in biology and medicine. The materials exhibited visible UC emission under 975-nm excitation, which confirmed the efficient energy transfer between the used dopant ions. 


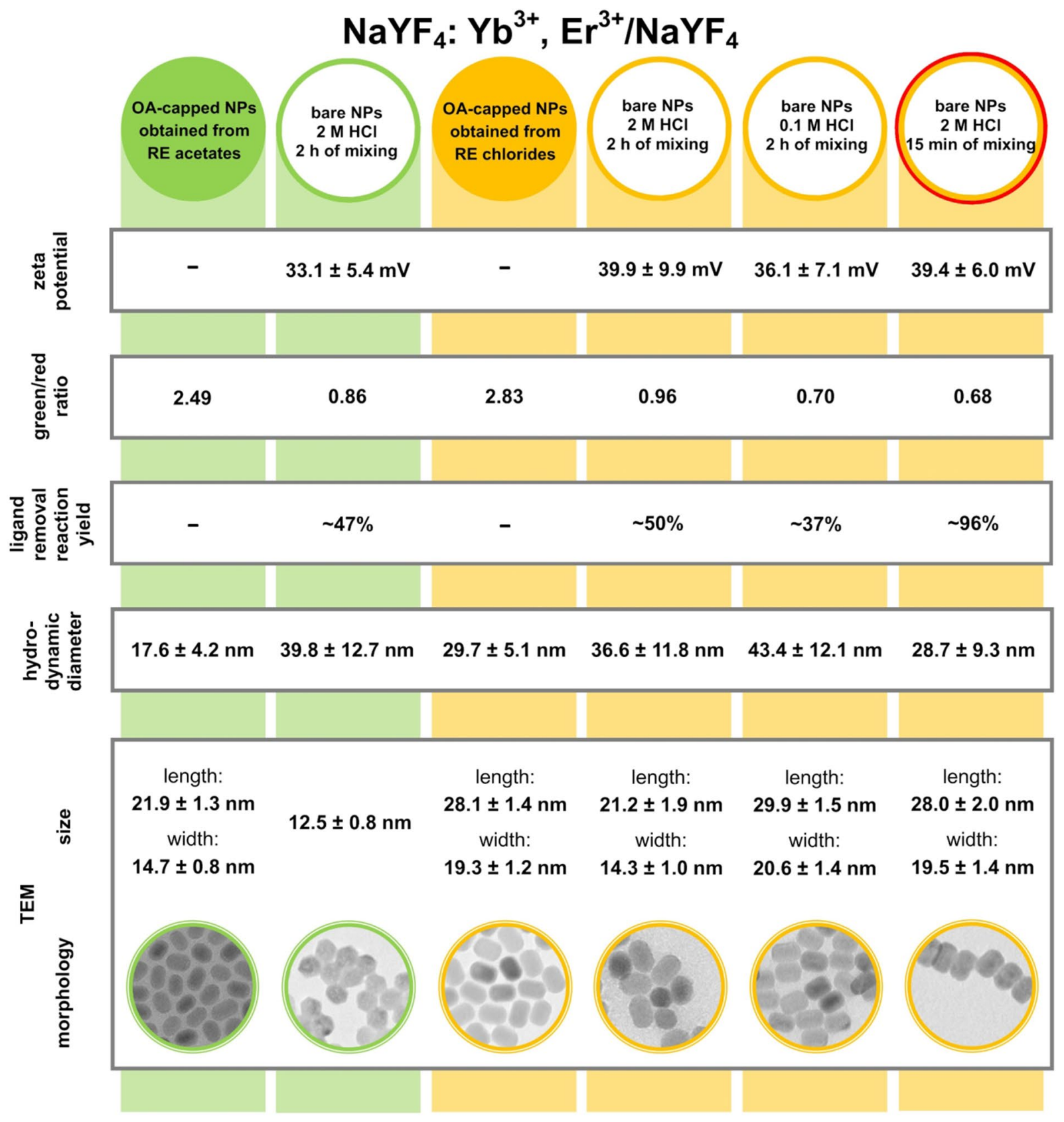

Figure 6. Overview of the zeta potential measurements, green-to-red emission ratios, surface modification yields, DLS measurements, and TEM analyses for both the OA-capped and the bare $\mathrm{NaYF}_{4}: \mathrm{Yb}^{3+}, \mathrm{Er}^{3+} / \mathrm{NaYF}_{4}$ particles. Color coding is used to indicate the UCNPs obtained from the RE acetates (green) and the RE chlorides (orange). The ligand-free product with the best properties was marked with the additional red circle.

Received: 2 April 2021; Accepted: 6 September 2021

Published online: 22 September 2021

\section{References}

1. Yi, Z., Luo, Z., Qin, X., Chen, Q. \& Liu, X. Lanthanide-activated nanoparticles: A toolbox for bioimaging, therapeutics, and neuromodulation. Acc. Chem. Res. 53, 2692-2704 (2020).

2. Auzel, F. Upconversion and anti-stokes processes with $\mathrm{f}$ and $\mathrm{d}$ ions in solids. Chem. Rev. 104, 139-173 (2004).

3. DaCosta, M. V., Doughan, S., Han, Y. \& Krull, U. J. Lanthanide upconversion nanoparticles and applications in bioassays and bioimaging: A review. Anal. Chim. Acta 832, 1-33 (2014).

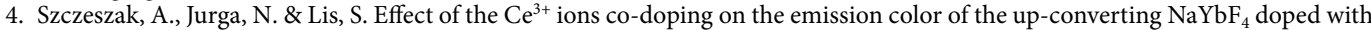
$\mathrm{Ho}^{3+}$ ions. Ceram. Int. 46, 26382-26387 (2020).

5. Przybylska, D., Ekner-Grzyb, A., Grześkowiak, B. F. \& Grzyb, T. Upconverting $\mathrm{SrF}_{2}$ nanoparticles doped with $\mathrm{Yb}^{3+} / \mathrm{Ho}^{3+}, \mathrm{Yb}^{3+} /$ $\mathrm{Er}^{3+}$ and $\mathrm{Yb}^{3+} / \mathrm{Tm}^{3+}$ ions-Optimisation of synthesis method, structural, spectroscopic and cytotoxicity studies. Sci. Rep. 9, 8669 (2019). 
6. Zhang, Z., Rahmat, J. N., Mahendran, R. \& Zhang, Y. Controllable assembly of upconversion nanoparticles enhanced tumor cell penetration and killing efficiency. Adv. Sci. 7, 1-12 (2020).

7. Huy, B. T., Kumar, A. P., Thuy, T. T., Nghia, N. N. \& Lee, Y. I. Recent advances in fluorescent upconversion nanomaterials: Novel strategies for enhancing optical and magnetic properties to biochemical sensing and imaging applications. Appl. Spectrosc. Rev. 50, 5809-5829 (2020).

8. Haase, M. \& Schäfer, H. Upconverting nanoparticles. Angew. Chem. Int. Ed. 50, 5808-5829 (2011).

9. Rabouw, F. T. et al. Quenching pathways in $\mathrm{NaYF}_{4}: \mathrm{Er}^{3+}, \mathrm{Yb}^{3+}$ upconversion nanocrystals. ACS Nano 12, 4812-4823 (2018).

10. Hong, E. et al. Control synthesis, subtle surface modification of rare-earth-doped upconversion nanoparticles and their applications in cancer diagnosis and treatment. Mater. Sci. Eng. C 105, 110097 (2019).

11. Sedlmeier, A. \& Gorris, H. H. Surface modification and characterization of photon-upconverting nanoparticles for bioanalytical applications. Chem. Soc. Rev. 44, 1526-1560 (2015).

12. Heuer-Jungemann, A. et al. The role of ligands in the chemical synthesis and applications of inorganic nanoparticles. Chem. Rev. $119,4819-4880$ (2019).

13. Duan, C., Liang, L., Li, L., Zhang, R. \& Xu, Z. P. Recent progress in upconversion luminescence nanomaterials for biomedical applications. J. Mater. Chem. B 6, 192-207 (2018).

14. Chen, X. et al. NIR-to-red upconversion nanoparticles with minimized heating effect for synchronous multidrug resistance tumor imaging and therapy. ACS Appl. Mater. Interfaces 10, 14378-14388 (2018).

15. Liang, G. et al. Recent progress in the development of upconversion nanomaterials in bioimaging and disease treatment. J. Nanobiotechnol. 18, 1-22 (2020).

16. Li, X., Yi, Z., Xue, Z., Zeng, S. \& Liu, H. Multifunctional $\mathrm{BaYbF}_{5}$ : Gd/Er upconversion nanoparticles for in vivo tri-modal upconversion optical, X-ray computed tomography and magnetic resonance imaging. Mater. Sci. Eng. C 75, 510-516 (2017).

17. Kumar, B., Murali, A., Mattan, I. \& Giri, S. Near-infrared-triggered photodynamic, photothermal, and on demand chemotherapy by multifunctional upconversion nanocomposite. J. Phys. Chem. B 123, 3738-3755 (2019).

18. Zhang, Y. et al. Near-infrared-triggered antibacterial and antifungal photodynamic therapy based on lanthanide-doped upconversion nanoparticles. Nanoscale 10, 15485-15495 (2018).

19. Yan, Q. et al. Lanthanide-doped nanoparticles encountering porphyrin hydrate: Boosting a dual-mode optical nanokit for $\mathrm{Cu}^{2+}$ sensing. Sens. Actuators B Chem. 268, 108-114 (2018).

20. Hemmer, E., Quintanilla, M., Légaré, F. \& Vetrone, F. Temperature-induced energy transfer in dye-conjugated upconverting nanoparticles: A new candidate for nanothermometry. Chem. Mater. 27, 235-244 (2015).

21. Guo, S., Xie, X., Huang, L. \& Huang, W. Sensitive water probing through nonlinear photon upconversion of lanthanide-doped nanoparticles. ACS Appl. Mater. Interfaces 8, 847-853 (2016).

22. Gnach, A. \& Bednarkiewicz, A. Lanthanide-doped up-converting nanoparticles: Merits and challenges. Nano Today 7, 532-563 (2012).

23. Bogdan, N., Vetrone, F., Ozin, G. A. \& Capobianco, J. A. Synthesis of ligand-free colloidally stable water dispersible brightly luminescent lanthanide-doped upconverting nanoparticles. Nano Lett. 11, 835-840 (2011).

24. Himmelstoß, S. F. \& Hirsch, T. Long-term colloidal and chemical stability in aqueous media of $\mathrm{NaYF}_{4}$-type upconversion nanoparticles modified by ligand-exchange. Part. Part. Syst. Charact. 36, 1900235 (2019).

25. Bogdan, N. et al. Bio-functionalization of ligand-free upconverting lanthanide doped nanoparticles for bio-imaging and cell targeting. Nanoscale 4, 3647-3650 (2012).

26. Zeng, S. et al. Dual-modal upconversion fluorescent/X-ray imaging using ligand-free hexagonal phase $\mathrm{NaLuF}_{4}: \mathrm{Gd} / \mathrm{Yb} / \mathrm{Er}$ nanorods for blood vessel visualization. Biomaterials 35, 2934-2941 (2014).

27. Liu, N. et al. Water dispersible ligand-free rare earth fluoride nanoparticles: water transfer versus $\mathrm{NaREF}_{4}$-to- $\mathrm{REF}_{3}$ phase transformation. Dalton. Trans. 49, 16204-16216 (2020).

28. Hong, A. R. et al. Intense red-emitting upconversion nanophosphors ( $800 \mathrm{~nm}$-driven) with a core/double-shell structure for dual-modal upconversion luminescence and magnetic resonance in vivo imaging applications. ACS Appl. Mater. Interfaces 10, 12331-12340 (2018).

29. Wu, B., Cao, Z., Zhang, Q. \& Wang, G. NIR-responsive DNA hybridization detection by high efficient FRET from 10-nm upconversion nanoparticles to SYBR green I. Sens. Actuators B Chem. 255, 2853-2860 (2018).

30. Gerelkhuu, Z. et al. One-step synthesis of $\mathrm{NaLu}_{80-\mathrm{x}} \mathrm{Gd}_{\mathrm{x}} \mathrm{F}_{4}: \mathrm{Yb}_{18}{ }^{3+} / \mathrm{Er}_{2}{ }^{3+}\left(\mathrm{Tm}^{3+}\right)$ upconversion nanoparticles for in vitro cell imaging. Mater. Sci. Eng. C 86, 56-61 (2018).

31. Rinkel, T., Raj, A. N., Dühnen, S. \& Haase, M. Synthesis of $10 \mathrm{~nm} \beta-\mathrm{NaYF}_{4}: \mathrm{Yb}, \mathrm{Er} / \mathrm{NaYF}_{4}$ core/shell upconversion nanocrystals with $5 \mathrm{~nm}$ particle cores. Angew. Chem. 128, 1177-1181 (2016).

32. Kuk, S. K. et al. Siloxane-encapsulated upconversion nanoparticle hybrid composite with highly stable photoluminescence against heat and moisture. ACS Appl. Mater. Interfaces 11, 15952-15959 (2019).

33. Lisjak, D., Plohl, O., Ponikvar-Svet, M. \& Majaron, B. Dissolution of upconverting fluoride nanoparticles in aqueous suspensions. RSC Adv. 5, 27393-27397 (2015).

34. McIntosh, I. M., Nichols, A. R. L., Tani, K. \& Llewellin, E. W. Accounting for the species-dependence of the $3500 \mathrm{~cm}^{-1} \mathrm{H}_{2} \mathrm{O}_{\mathrm{t}}$ infrared molar absorptivity coefficient: Implications for hydrated volcanic glasses. Am. Mineral. 102, 1677-1689 (2017).

35. Würth, C. et al. Excitation power dependent population pathways and absolute quantum yields of upconversion nanoparticles in different solvents. Nanoscale 9, 4283-4294 (2017).

36. Scheps, R. Upconversion laser processes. Prog. Quantum Electron. 20, 271-358 (1996).

37. Lei, Y. et al. Upconversion luminescence, intensity saturation effect, and thermal effect in $\mathrm{Gd}_{2} \mathrm{O}_{3}: \mathrm{Er}^{3+}, \mathrm{Yb}^{3+}$ nanowires. J. Chem. Phys. 123, 174710 (2005).

\section{Author contributions}

N.J. modified the surface of the nanoparticles and performed the DLS, zeta potential, and spectroscopic measurements. D.P and P.K. conducted the nanoparticle synthesis. N.J. and T.G. analyzed the obtained results. All of the authors wrote and reviewed the manuscript.

\section{Funding}

This research was funded by the National Science Center Poland (UMO-2016/22/E/ST5/00016) and Grant No. POWR.03.02.00-00-I020/17 co-financed by the European Union through the European Social Fund under the Operational Program Knowledge Education Development.

\section{Competing interests}

The authors declare no competing interests. 


\section{Additional information}

Supplementary Information The online version contains supplementary material available at https://doi.org/ 10.1038/s41598-021-98240-0.

Correspondence and requests for materials should be addressed to T.G.

Reprints and permissions information is available at www.nature.com/reprints.

Publisher's note Springer Nature remains neutral with regard to jurisdictional claims in published maps and institutional affiliations.

(c) (1) Open Access This article is licensed under a Creative Commons Attribution 4.0 International cc) License, which permits use, sharing, adaptation, distribution and reproduction in any medium or format, as long as you give appropriate credit to the original author(s) and the source, provide a link to the Creative Commons licence, and indicate if changes were made. The images or other third party material in this article are included in the article's Creative Commons licence, unless indicated otherwise in a credit line to the material. If material is not included in the article's Creative Commons licence and your intended use is not permitted by statutory regulation or exceeds the permitted use, you will need to obtain permission directly from the copyright holder. To view a copy of this licence, visit http://creativecommons.org/licenses/by/4.0/.

(C) The Author(s) 2021 